TRANSACTIONS OF THE

AMERICAN MATHEMATICAL SOCIETY

Volume 363, Number 9, September 2011, Pages 4831-4851

S 0002-9947(2011)05258-X

Article electronically published on April 20, 2011

\title{
RECONSTRUCTING POTENTIALS FROM ZEROS OF ONE EIGENFUNCTION
}

\author{
XINFU CHEN, Y. H. CHENG, AND C. K. LAW
}

\begin{abstract}
We study an inverse nodal problem, concerning the reconstruction of a potential of a Sturm-Liouville operator, by using zeros of one eigenfunction as input. We propose three methods for the reconstruction, one of which is the Tikhonov regularization method. The explicit error bounds are calculated for all three methods. In case there is measurement error, the Tikhonov regularization method is still convergent. The study is motivated by physical considerations.
\end{abstract}

\section{INTRODUCTION}

In this paper, we are concerned about the reconstruction of a potential function

$$
q \in \mathbf{Q}:=\left\{p \in H^{1}([0,1]) \mid \int_{0}^{1} p(x) d x=0\right\}
$$

from the set of zeros of an eigenfunction to the one-dimensional Schrödinger equation

$$
\left\{\begin{array}{l}
\ddot{u}=(q-\lambda) u \text { in }(0,1), \\
u(0)=u(1)=0
\end{array}\right.
$$

where the dots denote differentiation. Denote by

$$
\left\{\lambda_{n}, u_{n}\right\}_{n=1}^{\infty}=\left\{(\lambda(n, q), u(n, q, \cdot)\}_{n=1}^{\infty}\right.
$$

all the eigenpairs of (1.1), where $\lambda_{n}<\lambda_{n+1}$ for all $n \geq 1$. Then the set

$$
\left\{x \in[0,1] \mid u_{n}(x)=0\right\}=\left\{z_{0}, z_{1}, \cdots, z_{n}\right\}
$$

has exactly $n+1$ elements and depends only on $q$. We shall also normalize the eigenfunctions $u_{n}$ by $\dot{u}_{n}(0)=1$. We use the notation

$$
\begin{aligned}
\mathbf{z}(n, q) & :=\left(z_{0}, \cdots, z_{n}\right) \in \mathbf{X}(n), \\
\mathbf{X}(n) & :=\left\{\left(x_{0}, \cdots, x_{n}\right) \in \mathbb{R}^{n+1} \mid 0=x_{0}<x_{1}<\cdots<x_{n-1}<x_{n}=1\right\} .
\end{aligned}
$$

We call each $\mathbf{z}(n, q)(n=1,2, \cdots)$ a nodal set of $q$, whereas the coordinates of $\mathbf{z}$ will be regarded as points in $[0,1]$.

In many applications certain nodal set(s) of a potential can be measured. It would be very helpful if one can calculate the potential (inhomogeneity) from the

Received by the editors November 4, 2008 and, in revised form, November 18, 2009.

2010 Mathematics Subject Classification. Primary 34B24, 47A52; Secondary 34A55, 49M30.

Key words and phrases. Inverse nodal problem, Sturm-Liouville operators, Tikhonov regularization, error bound. 
nodal set(s). In general, one may measure a set $\left\{x_{0}, \cdots, x_{n}\right\}$ of zeros of an oscillatory solution $u$ to $\ddot{u}=V u$ in an interval $\left[x_{0}, x_{n}\right] \subset \mathbb{R}$ and want to calculate the potential $V$ restricted on $\left[x_{0}, x_{n}\right]$. After a linear scaling that maps $x_{0}$ to 0 and $x_{n}$ to $1, u$ will be a solution to (1.1) with $q=V-\bar{V}$ and $\lambda=-\bar{V}$, where the bar stands for average. It is then natural to solve the following.

Problem 1. Given positive integers $\left\{n_{i}\right\}_{i=1}^{m}$ where $1 \leqslant n_{1}<\cdots<n_{m}$ and sets $\mathbf{x}\left(n_{i}\right) \in \mathbf{X}\left(n_{i}\right), i=1, \cdots, m$, find $q \in \mathbf{Q}$ such that its nodal set $\mathbf{z}\left(n_{i}, q\right)=\mathbf{x}\left(n_{i}\right)$ for every $i=1, \cdots, m$.

Such a problem is nonlinear and is often called the inverse nodal problem. A typical one involves the reconstruction of coefficients $p, \rho$ or $q$ of the Sturm-Liouville type operators

$$
\begin{aligned}
\mathcal{L}_{1}[\lambda, u]=\left(p u^{\prime}\right)^{\prime}+\lambda u, & \mathcal{L}_{2}[\lambda, u] & =u^{\prime \prime}+\lambda \rho u, \\
\mathcal{L}_{3}[\lambda, u]=u^{\prime \prime}+(\lambda-q) u, & \mathcal{L}_{4}[\lambda, u] & =\left(p u^{\prime}\right)^{\prime}+\lambda \rho u
\end{aligned}
$$

from the zeros of its eigenfunction(s), referred to as the nodal data. Inverse nodal problems were first studied by McLaughlin 11 and Shen 12. Many reconstruction formulas have since been derived and analyzed; see, for example, Hald-McLaughlin [5] and Law-Shen-Yang [8]. In particular, in [5], Hald-McLaughlin provided, among others, two numerical algorithms for the reconstruction of $q$. One of the algorithms can be induced from (1.4) below, while the other needs the information about the eigenvalues as well and so is not of our interest for this paper.

For a physicist, solving Problem 1 has many potential applications and therefore is quite important; for a pure mathematician, the problem can be considered as solved since many uniqueness and reconstruction results have been proved [3, 4, 5, 6. 10, 8, 9, 11, 12, 13, 14. However, from an applied point of view, there are still a number of issues to be resolved.

First of all, the problem could be over-determined; namely, given a sequence $\left\{\mathbf{x}\left(n_{i}\right)\right\}_{i=1}^{\infty}$, there may not exist a potential $q$ such that $\mathbf{x}\left(n_{i}\right)=\mathbf{z}\left(q, n_{i}\right)$ for all $i$, although it is well known that such a potential, if it exists, is unique; see [9] and the references therein. Indeed, a very surprising result of Yang [14] (see also [4]) says that a dense subset of all the nodes in $\left(0, \frac{1}{2}+\epsilon\right)(\epsilon$ arbitrarily small but positive) is sufficient to determine $q$ over the whole interval $[0,1]$ (although no reconstruction formula is known so far to recover the part of the potential in $\left.\left(\frac{1}{2}+\epsilon, 1\right)\right)$. From this, it is easy to realize that the inverse problem is highly unstable if one intends to find a potential that matches exactly the given nodal data for a large number of eigenfunctions.

So far all the known reconstruction formulas rely on an accurate measurement of the nodal data; in other words, the potential sensitively depends on the nodal set. Indeed, to calculate $q$ from $\mathbf{x}(n)=\left\{x_{0}, \cdots, x_{n}\right\}$, the accuracy needed for each point in $\mathbf{x}(n)$ should be of order $o(1) n^{-3}$. This can be seen from the asymptotic expansion, assuming that $q$ is smooth,

$$
x_{k+1}-x_{k}=\frac{1}{n}+\frac{q\left(x_{k+1}\right)+q\left(x_{k}\right)}{4 \pi^{2} n^{3}}+\frac{O(1)}{n^{5}} \quad \forall k=0, \cdots, n-1 .
$$

For example, suppose $n=100$. If the position of $x_{10}$ is measured as 0.1000001 instead of 0.1000000 , then it will produce an error of $10^{-7} \times 4 \pi^{2} n^{3} \approx 4$ toward the potential; see Figure 1. That is to say, in deriving various kinds of asymptotic formulas (as $n \rightarrow \infty$ ) for the reconstruction of $q$ (and its derivatives), one has to pay 
attention to the physical requirement on the accuracy needed for the measurement of the nodal set.

We remark that (1.2), to be proven at the end of this paper, is indeed the core of many known reconstruction formulas.

In practice, the problem of possible over-determination can be solved by restricting the number of nodal sets used. As a start, in this paper we shall take only one nodal set (i.e., $m=1$ in Problem 1). We shall introduce a technique that can handle, in a certain degree, the possible noises introduced in the measurement. Though the problem is not completely solved in this paper, the main goal here is to introduce a new direction that deserves attention in the study of inverse nodal problems.

For the reader's convenience, we provide a few more details about the Inverse Problem 1.

1. When $m=1$, the problem is always solvable. For example, given $n \geqslant 1$ and $\mathbf{x}=\left(x_{0}, \cdots, x_{n}\right) \in \mathbf{X}(n)$, we can obtain a potential $q=P$ of the form

$P=V+\lambda, \quad \lambda=-\int_{0}^{1} V(x) d x, \quad V(x)=\zeta_{0}(x)-\sum_{i=1}^{n} \gamma_{i} \zeta_{i}(x) \chi_{\left[x_{i-1}, x_{i}\right)}(x)$,

where $\zeta_{0}, \cdots, \zeta_{n}$ are arbitrarily chosen nontrivial and nonnegative functions, and $\gamma_{1}, \cdots, \gamma_{n}$ are parameters to be determined from a system of $n$ algebraic equations. Here and in the sequel, $\chi_{A}$ is the characteristic function of the set $A$, i.e.,

$$
\chi_{A}(x):= \begin{cases}1 & \text { if } x \in A, \\ 0 & \text { if } x \notin A .\end{cases}
$$

In particular, an explicit solution is given by $q=P$, where

$$
V=-\sum_{i=0}^{n-1}\left(\frac{\pi}{x_{i+1}-x_{i}}\right)^{2} \chi_{\left[x_{i}, x_{i+1}\right)}, \quad \lambda=\sum_{i=0}^{n-1} \frac{\pi^{2}}{x_{i+1}-x_{i}} .
$$

2. When $m \geqslant 2$, it follows from the Sturm comparison theorem that the following necessary condition is needed:

For each $1 \leqslant i<j \leqslant m$, between any neighboring points in $\mathbf{x}\left(n_{i}\right)$, there is at least one point in $\mathbf{x}\left(n_{j}\right)$.

It is our conjecture that such a condition is also sufficient for Problem 1 to be solvable.

3. Suppose $m=\infty$. In a series of works $[8,9,10$, it is shown that a potential, if it exists, is unique and can be obtained from the following limit: writing $\mathbf{x}(n)=$ $\left(x_{0}^{(n)}, \cdots, x_{n}^{(n)}\right) \in \mathbf{X}(n)$ for $n=n_{1}, n_{2}, \cdots$,

$$
q(x)=\lim _{n \rightarrow \infty} 2 \pi^{2} n^{2}\left\{n L_{n}(x)-1\right\}, \quad L_{n}:=\sum_{i=0}^{n-1}\left(x_{i+1}^{(n)}-x_{i}^{(n)}\right) \chi_{\left[x_{i}^{(n)}, x_{i+1}^{(n)}\right)} .
$$

We remark that in [8, 9, 10], the function $L_{n}$ was expressed as

$$
L_{n}(x):=x_{j+1}^{(n)}-x_{j}^{(n)}, \quad \text { where } j=j_{n}(x):=\max \left\{k \mid x_{k}^{(n)} \leq x\right\} .
$$

Here we use one of its equivalent alternatives.

One also observes that in using the above limit for the reconstruction of a reliable $q$, a sufficient accuracy is needed for the measurement of the nodal set $\mathbf{x}(n)$; namely, the error of measurement should be controlled at an order of $o(1) n^{-3}$, where $o(1)$ is small. 
There are many ways to modify the formula (1.4) so that the needed accuracy of measurement can be relaxed. For example, one can count zeros by groups; that is, for some $0=k_{0}<k_{1}<\cdots<k_{J}=n$, replace $L_{n}$ by

$$
\tilde{L}_{n}=\sum_{j=1}^{J} \frac{x_{k_{j}}^{(n)}-x_{k_{j-1}}^{(n)}}{k_{j}-k_{j-1}} \chi_{\left[x_{k_{j-1}}^{(n)}, x_{k_{j}}^{(n)}\right)} .
$$

We shall provide error estimates for the reconstruction formulas (1.3), (1.4), and (1.5). Furthermore we shall focus on the following:

Problem 2. Design an algorithm $\mathcal{A}$ that produces an output $P=\mathcal{A}(n, \mathbf{x})$ for each input consisting of a positive integer $n$ and a nodal set $\mathbf{x} \in \mathbf{X}(n)$.

Surely, we hope that the algorithm has the property that if $\hat{\mathbf{x}}=\mathbf{z}(n, q)$ and $\mathbf{x}$ is close to $\hat{\mathbf{x}}$, then $P=\mathcal{A}(n, \mathbf{x})$ is close to $q$ in a certain sense. To do this, here in this paper we propose to use the Tikhonov regularization method (cf. [7] for example). Fixing a small positive constant $\varepsilon>0$, we define a Tikhonov functional on $\mathbf{X}(n) \times \mathbf{Q}$ by

$$
\mathbf{E}(n, \varepsilon, \mathbf{x}, p):=\frac{|\mathbf{x}-\mathbf{z}(n, p)|^{2}}{\varepsilon}+\int_{0}^{1} \dot{p}^{2}(x) d x \quad \forall \mathbf{x} \in \mathbf{X}(n), p \in \mathbf{Q},
$$

where $|\cdot|$ is the Euclidean distance in $\mathbb{R}^{n+1}$. Consider the following minimization problem.

Problem 3. Given a real $\varepsilon>0$, an integer $n \geqslant 1$ and $\mathbf{x} \in \mathbf{X}(n)$, find $p_{\varepsilon}$ such that

$$
p_{\varepsilon} \in \mathbf{Q}, \quad \mathbf{E}\left(n, \varepsilon, \mathbf{x}, p_{\varepsilon}\right)=\min _{p \in \mathbf{Q}} \mathbf{E}(n, \varepsilon, \mathbf{x}, p) .
$$

Other choices of Tikhonov functionals can also be used. For example,

$$
\mathbf{E}_{1}(n, \varepsilon, \mathbf{x}, p):=\frac{|\delta \mathbf{x}-\delta \mathbf{z}(n, p)|^{2}}{\varepsilon}+\int_{0}^{1} \dot{p}^{2}(x) d x \quad \forall \mathbf{x} \in \mathbf{X}(n), p \in \mathbf{Q} .
$$

Here and in the sequel, we use the following notation: for $\mathbf{y}=\left(y_{0}, y_{1}, \cdots, y_{n}\right) \in$ $\mathbb{R}^{n+1}$

$$
\begin{gathered}
\delta y_{i}:=y_{i+1}-y_{i}, \quad \delta^{2} y_{i}:=y_{i+1}+y_{i-1}-2 y_{i}, \\
\delta \mathbf{y}:=\left(\delta y_{0}, \cdots, \delta y_{n-1}\right) \in \mathbb{R}^{n}, \quad \delta^{2} \mathbf{y}:=\left(\delta^{2} y_{1}, \cdots, \delta^{2} y_{n-1}\right) \in \mathbb{R}^{n-1} .
\end{gathered}
$$

When $\varepsilon=0$, both $\mathbf{E}$ and $\mathbf{E}_{1}$ reduce to the functional

$$
\mathbf{E}_{2}(p):=\|\dot{p}\|^{2}:=\int_{0}^{1} \dot{p}^{2}(x) d x, \quad p \in \mathbf{Q}^{\mathbf{x}}:=\{q \in \mathbf{Q} \mid \mathbf{z}(n, q)=\mathbf{x}\} .
$$

Our main results are the following.

Theorem 1.1. Given $\mathbf{x} \in \mathbf{X}(n)$, define $P$ as in (1.3) and $L_{n}$ as in (1.4) or (1.5). If $q \in \mathbf{Q}$ satisfies $\mathbf{z}(n, q)=\mathbf{x}$ and $\int_{0}^{1}|q(x)| d x<n \pi^{2} / 4$, then, for all $r \geq 1$,

$$
\begin{aligned}
\|q-P\|_{L^{r}} & \leqslant 4\|q-\tilde{q}\|_{L^{r}}, \\
\left\|2 \pi^{2} n^{2}\left(n L_{n}-1\right)-q\right\|_{L^{r}} & \leqslant \frac{10}{3}\|q-\tilde{q}\|_{L^{r}}+\frac{31 \max _{k} \overline{|q|}_{k}}{2 n^{2} \pi^{2}}\|q\|_{L^{r}},
\end{aligned}
$$

where $L^{r}=L^{r}((0,1))$ is the $L^{r}$-norm,

$$
\bar{q}_{k}:=\frac{1}{\delta x_{k}} \int_{x_{k}}^{x_{k+1}} q(x) d x, \quad \overline{|q|}_{k}:=\frac{1}{\delta x_{k}} \int_{x_{k}}^{x_{k+1}}|q(x)| d x,
$$


and $\tilde{q}=\sum_{k=1}^{n} \bar{q}_{k} \chi_{\left[x_{k-1}, x_{k}\right)}$ is the projection of $q$ over the set of piecewise constant functions.

A similar estimate for $\tilde{L}_{n}$ defined in (1.5) will also be provided.

For the Tikhonov regularization method, we shall prove the following:

Theorem 1.2. (a) For any $\mathbf{x} \in \mathbf{X}(n)$ and $\varepsilon>0$, there is at least a minimizer $p_{\varepsilon}$ of $\mathbf{E}(n, \varepsilon, \mathbf{x} ; \cdot)$.

(b) If $q \in \mathbf{Q}$ satisfies $\mathbf{x}=\mathbf{z}(n, q)$ and $\int_{0}^{1}|\dot{q}|^{2} \leq\left(\frac{\pi}{2}\right)^{6} n^{2}$, then

$$
\left\|p_{\varepsilon}-q\right\|^{2} \leq C\left(\frac{1}{n^{2}}+\varepsilon n^{5}\right) \int_{0}^{1} \dot{q}^{2} d x .
$$

Here $C$ is a universal constant.

(c) Furthermore if $q^{\alpha} \in \mathbf{Q}$ satisfies $\mathbf{x}^{\alpha}=\mathbf{z}\left(n, q^{\alpha}\right)$ with $\left\|x-x^{\alpha}\right\|<\alpha$, and $\int_{0}^{1}\left|\dot{q}^{\alpha}\right|^{2} \leq\left(\frac{\pi}{2}\right)^{6} n^{2}$, then $p_{\varepsilon}^{\alpha}$, the minimizer of $\mathbf{E}\left(n, \varepsilon, x^{\alpha}, \cdot\right)$, satisfies, with the universal constant $C$ given above,

$$
\left\|p_{\varepsilon}^{\alpha}-q\right\|^{2} \leq 2 C n^{5} \alpha^{2}+\frac{2 C}{n^{2}}\|\dot{q}\|^{2}+C\left(\frac{3}{n^{2}}+\varepsilon n^{5}\right)\left\|\dot{q}^{\alpha}\right\|^{2} .
$$

Remark 1.3. Hence if $\|\dot{q}\|^{2},\left\|\dot{q}^{\alpha}\right\|^{2}<M<\left(\frac{\pi}{2}\right)^{6} n^{2}$, and $\varepsilon<n^{-7}$, then

$$
\left\|p_{\varepsilon}-q\right\| \leq \frac{C M}{n^{2}} .
$$

However if we take into account the accuracy of the measurement and assume that $\left|x-x^{\alpha}\right|<\alpha$, then letting $n=\alpha^{-2 / 7}$, we have

$$
\begin{aligned}
\left\|p_{\varepsilon}^{\alpha}-q\right\|^{2} & \leq C\left(\frac{5}{n^{2}}+\varepsilon n^{5}\right) M+2 C n^{5} \alpha^{2} \\
& \leq 8 C M \alpha^{4 / 7}
\end{aligned}
$$

This shows the convergence of this Tikhonov regularization method when there is an error in the measurement of $\mathbf{x}$ for $\mathbf{z}(n, q)$.

Remark 1.4. The error estimate for $V$ is exactly the same using the same argument (cf. the proofs of Theorem 4.1 and Theorem 2). So

$$
\left\|V^{\alpha}-q\right\| \leq C n^{5} \alpha^{2}+\frac{C}{n^{2}}\left(\|\dot{q}\|^{2}+\left\|\dot{q}^{\alpha}\right\|^{2}\right) .
$$

Although currently we cannot show the uniqueness of solutions to our regularization method, it is expected that any solution will provide a reasonable potential $P$ for a target unknown $q$, even when there are errors in the measurement $\mathbf{x}$ for $\mathbf{z}(n, q)$. We illustrate our point of view by a numerical simulation demonstrated in Figure 1, In fact, the figure shows that the regularization method is even more accurate than the other methods. We shall pursue this in another paper.

In [2], Barnes also considered a variational formulation, in fact the least square method, in solving the inverse eigenvalue problem with a finite number of eigenvalues. There he used weaker topologies for $q$ instead of a penalty term. The existence of solutions was shown and numerical conditioning of the scheme was studied.

In Section 2, we shall show the existence of solutions to the minimizers of different Tikhonov functionals and derive the associated Euler-Lagrange equations. In Section 3, we shall prove Theorem 1.1 giving explicit error bounds for the schemes (1.3) and (1.5). A new modified Prüfer substitution is introduced for the analysis. 


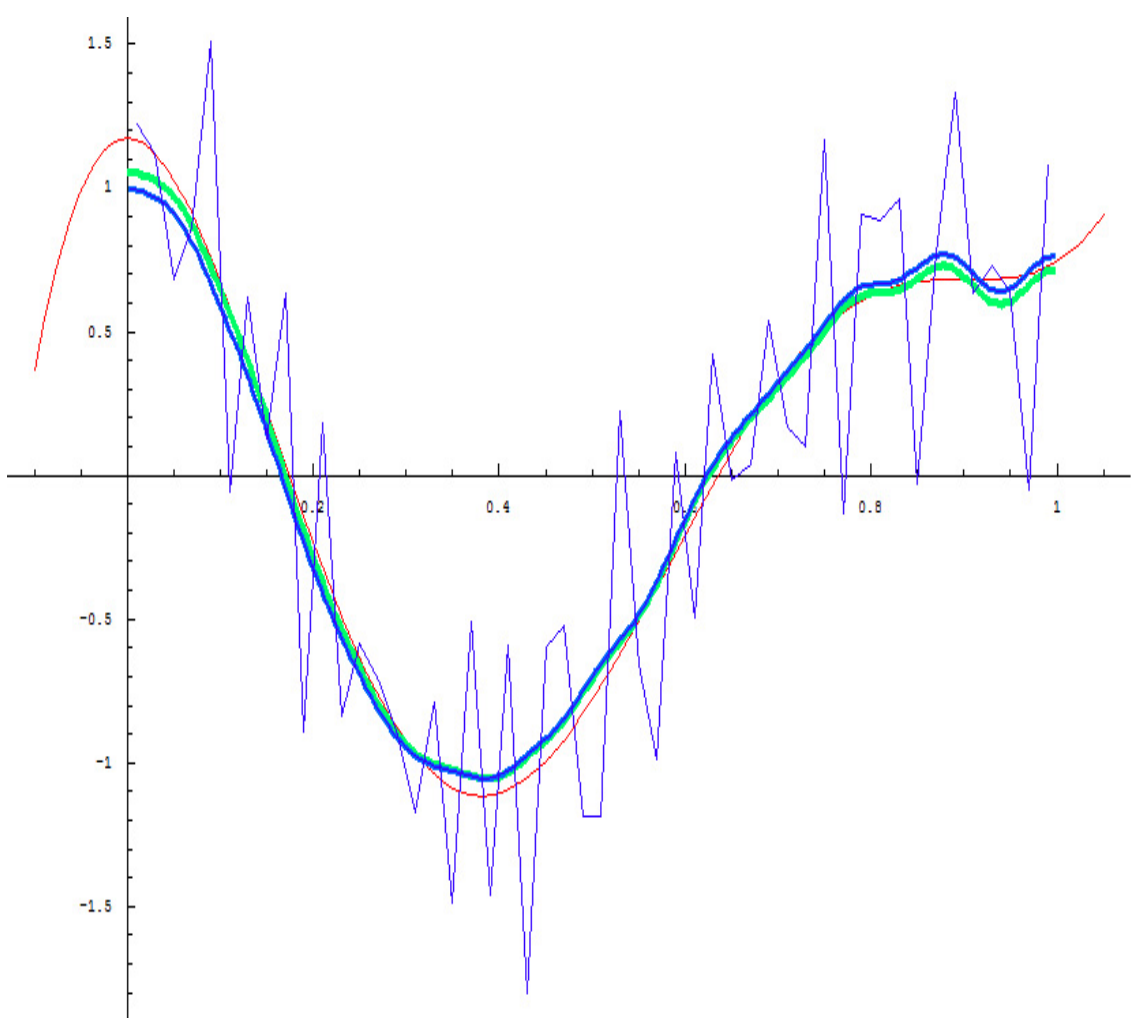

Figure 1. Comparison of the schemes: (i) The smooth curve is the true potential $p$; (ii) the thin zigzagged curve is a reconstruction using (1.4) from a noised measurement $\mathbf{x}$ of the true nodal set $\hat{\mathbf{x}}$ with random error of size $5 \times 10^{-8}$; (iii) the dark thick curve and light thick curve are reconstructions using the minimizers of $\mathbf{E}_{2}$ and $\mathbf{E}_{1}$ (overlapped), and $\mathbf{E}$, respectively, with the same noised measurement $\mathbf{x}$ as in (ii).

Finally, we shall have stability estimates in Section 4, culminating in the proof of Theorem 1.2. A proof of (1.2) will appear in the Appendix, again using the modified Prüfer substitution.

\section{The Tikhonov Regularization Method}

In this section, we briefly study the minimization problem for the Tikhonov functionals $\mathbf{E}, \mathbf{E}_{1}$ and $\mathbf{E}_{2}$. In particular, we prove the following:

Theorem 2.1. Given a positive integer $n$ and $\mathbf{x} \in \mathbf{X}(n), \mathbf{E}_{2}(\cdot)$ in $\mathbf{Q}^{\mathbf{x}}$ admits a minimizer and for every $\varepsilon>0$, both $\mathbf{E}(n, \varepsilon, \mathbf{x} ; \cdot)$ and $\mathbf{E}_{1}(n, \varepsilon, \mathbf{x} ; \cdot)$ in $\mathbf{Q}$ admit minimizers.

In addition, let $p_{\varepsilon}$ be a minimizer to $\mathbf{E}(n, \varepsilon, \mathbf{x} ; \cdot)$ or to $\mathbf{E}_{1}(n, \varepsilon, \mathbf{x} ; \cdot)$ in $\mathbf{Q}$. Then along a sequence of $\varepsilon \searrow 0, p_{\varepsilon} \longrightarrow p_{0}$, where $p_{0} \in \mathbf{Q}^{\mathbf{x}}$ is a minimizer of $\mathbf{E}_{2}(\cdot)$ in $\mathrm{Q}^{\mathrm{x}}$. 
2.1. Existence of a minimizer. We consider only the minimization problem for E. The same problems as those for $\mathbf{E}_{1}$ or $\mathbf{E}_{2}$ can be considered in a similar manner and are omitted.

For notational simplicity, we write $\mathbf{E}(p)=\mathbf{E}(n, \varepsilon, \mathbf{x} ; p)$. Since $\mathbf{E}$ is a nonnegative well-defined functional on $H^{1}([0,1]) \supset \mathbf{Q}$, there exists a minimizing sequence $\left\{p_{i}\right\}_{i=1}^{\infty}$, i.e.,

$$
p_{i} \in \mathbf{Q} \forall i, \quad \lim _{i \rightarrow \infty} \mathbf{E}\left(p_{i}\right)=\inf _{p \in \mathbf{Q}} \mathbf{E}(p) .
$$

This implies that $\left\{p_{i}\right\}$ is a bounded family in $H^{1}([0,1])$. So, by the weak compactness of any closed and bounded subset of $\mathbf{Q}$, there exists $p_{\varepsilon} \in \mathbf{Q}$ such that along a subsequence $j \rightarrow \infty$,

$$
p_{j} \quad \longrightarrow \quad p_{\varepsilon} \quad \text { weakly in } H^{1}((0,1)) \text { and uniformly in } C([0,1]) .
$$

Hence, by the weak lower semicontinuity of the functional $\int_{0}^{1} \dot{p}^{2}$, we have

$$
\int_{0}^{1} \dot{p}_{\varepsilon}^{2}(x) d x \leq \liminf _{j \rightarrow \infty} \int_{0}^{1} \dot{p}_{j}^{2}(x) d x
$$

Furthermore,

$$
\lim _{j \rightarrow \infty} \mathbf{z}\left(n, p_{j}\right)=\mathbf{z}\left(n, p_{\varepsilon}\right), \quad \lim _{j \rightarrow \infty} \lambda\left(n, p_{j}\right)=\lambda\left(n, p_{\varepsilon}\right),
$$

so that

$$
\mathbf{E}\left(p_{\varepsilon}\right) \leq \lim _{j \rightarrow \infty} \mathbf{E}\left(p_{j}\right)=\inf _{p \in \mathbf{Q}} \mathbf{E}(p) .
$$

Therefore, $p_{\varepsilon} \in \mathbf{Q}$ is a minimizer of $\mathbf{E}(n, \varepsilon, \mathbf{x} ; \cdot)$ in $\mathbf{Q}$.

2.2. The singular limit. For every $\varepsilon>0$, let $p_{\varepsilon}$ be a minimizer of $\mathbf{E}(n, \varepsilon, \mathbf{x} ; \cdot)$. We consider the asymptotic limit, as $\varepsilon \searrow 0$, of the minimizer $p_{\varepsilon}$.

Using the construction mentioned in Section 1, there exists $q \in \mathbf{Q}$ such that $\mathbf{x}=\mathbf{z}(n, q)$. It then follows that

$$
\mathbf{E}\left(n, \varepsilon, \mathbf{x} ; p_{\varepsilon}\right)=\frac{\left|\mathbf{x}-\mathbf{z}\left(n, p_{\varepsilon}\right)\right|^{2}}{\varepsilon}+\int_{0}^{1}\left|\dot{p}_{\varepsilon}\right|^{2} \leqslant \mathbf{E}(n, \varepsilon, \mathbf{x} ; q)=\int_{0}^{1}|\dot{q}|^{2} d x .
$$

Hence, using $\|\cdot\|$ for the $L^{2}((0,1))$ norm,

$$
\left\|\dot{p}_{\varepsilon}\right\| \leqslant\|\dot{q}\|, \quad\left|\mathbf{x}-\mathbf{z}\left(n, p_{\varepsilon}\right)\right|^{2} \leq \varepsilon\|\dot{q}\|^{2} \quad \forall \varepsilon>0 .
$$

Similar to the above, there exists $p_{0} \in \mathbf{Q}$ such that along a sequence $\varepsilon \searrow 0$,

$$
\begin{aligned}
p_{\varepsilon} & \longrightarrow p_{0} \quad \text { weakly in } H^{1}((0,1)) \text { and uniformly in } C([0,1]) \\
\mathbf{z}\left(n, p_{\varepsilon}\right) & \longrightarrow \mathbf{x}=\mathbf{z}\left(n, p_{0}\right) .
\end{aligned}
$$

Next we show that the limit $p_{0} \in \mathbf{Q}^{\mathbf{x}}$ is a minimizer of $\mathbf{E}_{2}$ in $\mathbf{Q}^{\mathbf{x}}$. Indeed, for any $q \in \mathbf{Q}$ satisfying $\mathbf{z}(n, q)=\mathbf{x}$, from the previous step, we see that $\left\|\dot{p}_{\varepsilon}\right\| \leq\|\dot{q}\|$, so

$$
\left\|\dot{p}_{0}\right\| \leq \liminf _{\varepsilon \rightarrow 0}\left\|\dot{p}_{\varepsilon}\right\| \leqslant\|\dot{q}\|
$$

Thus, $p_{0}$ is a minimizer of $\mathbf{E}_{2}$ in $\mathbf{Q}^{\mathbf{x}}$.

In a similar manner, one can study the asymptotic limit of minimizers of $\mathbf{E}_{1}(n, \varepsilon, \mathbf{x} ; \cdot)$. We only point out the following: for every positive integer $n$ and $\mathbf{x}, \mathbf{y} \in \mathbf{X}(n)$,

$$
2|\mathbf{x}-\mathbf{y}| \sin \frac{\pi}{2 n} \leqslant|\delta \mathbf{x}-\delta \mathbf{y}| \leqslant 2|\mathbf{x}-\mathbf{y}| \cos \frac{\pi}{2 n}
$$


2.3. The Euler-Lagrange equation. Here we shall derive the Euler-Lagrange equation for a minimizer. For this, we recall a well-known result from the elliptic pde theory: Suppose $f \in L^{r}((0,1))(r \geq 1)$ and $q \in H^{1}([0,1])$. Then

$$
\int_{0}^{1}(\dot{q} \dot{\zeta}+\zeta f) d x=0 \forall \zeta \in C^{\infty}([0,1]) \Longleftrightarrow\left\{\begin{array}{l}
\ddot{q}=f \quad \text { in } L^{r}((0,1)), \\
\dot{q}(0)=\dot{q}(1)=0 .
\end{array}\right.
$$

Now let $\varepsilon>0$, integer $n \geq 1$, and $\mathbf{x}=\left(x_{0}, \cdots, x_{n}\right) \in \mathbf{X}(n)$ be given and fixed. We write $\mathbf{E}(\cdot)=\mathbf{E}(n, \varepsilon, \mathbf{x} ; \cdot)$. For $p_{\varepsilon} \in \mathbf{Q}$ and $\zeta \in H^{1}((0,1))$, we want to calculate the first variation of $\mathbf{E}(\cdot)$ at $p_{\varepsilon}$ in the direction $\zeta-\bar{\zeta} \in \mathbf{Q}$, where $\bar{\zeta}=\int_{0}^{1} \zeta(x) d x$; namely, we calculate

$$
\left\langle\nabla \mathbf{E}\left(p_{\varepsilon}\right), \zeta-\bar{\zeta}\right\rangle:=\lim _{t \rightarrow 0} \frac{\mathbf{E}\left(p_{\varepsilon}+t[\zeta-\bar{\zeta}]\right)-\mathbf{E}\left(p_{\varepsilon}\right)}{t} .
$$

1. For $t \in \mathbb{R}$, set $p(t)=p_{\varepsilon}+t[\zeta-\bar{\zeta}], E(t)=\mathbf{E}(p(t)), Z(t)=\left(Z_{0}(t), \cdots, Z_{n}(t)\right):=$ $\mathbf{z}(n, p(t)), \Lambda(t)=\lambda(n, p(t))$ and $U(t, \cdot)=u(n, p(t) ; \cdot)$. Using the notation ${ }^{\prime}=\frac{d}{d t}$ and ${ }^{\circ}=\frac{d}{d x}$, we also let $V(t, \cdot):=U^{\prime}(t, \cdot)$. Note that $Z(0)=\mathbf{z}\left(n, p_{\varepsilon}\right), \Lambda(0)=\lambda$ and $U(0, \cdot)=u(\cdot)$.

From the definition of $\mathbf{E}$, we see that

$$
\left\langle\nabla \mathbf{E}\left(p_{\varepsilon}\right), \zeta-\bar{\zeta}\right\rangle=E^{\prime}(0), \quad E^{\prime}(t)=\frac{2(Z-\mathbf{x}) \cdot Z^{\prime}}{\varepsilon}+2 \int_{0}^{1}\left(\dot{p}_{\varepsilon}+t \dot{\zeta}\right) \dot{\zeta} d x
$$

Also, differentiating $U\left(t, Z_{i}(t)\right)=0$ in $t$, we obtain

$$
Z_{i}^{\prime}(t)=-\frac{V\left(t, Z_{i}(t)\right)}{\dot{U}\left(t, Z_{i}(t)\right)} \forall i
$$

2. Differentiating the equation $\ddot{U}=(p-\Lambda) U$ with respect to $t$ gives

$$
\ddot{V}=(p-\Lambda) V+\left(\zeta-\bar{\zeta}-\Lambda^{\prime}\right) U \text { in }(0,1), \quad V(t, 0)=V(t, 1)=0 .
$$

Note that the Wronskian $W=\dot{U} V-\dot{V} U$ satisfies $\dot{W}=-\left(\zeta-\bar{\zeta}-\Lambda^{\prime}\right) U^{2}$, so that

$$
\dot{U} V-\dot{V} U=-\int_{0}^{x}\left\{\zeta-\bar{\zeta}-\Lambda^{\prime}\right\} U^{2} .
$$

The conditions $U(t, 1)=V(t, 1)=0$ give

$$
\Lambda^{\prime}=-\bar{\zeta}+\frac{\int_{0}^{1} \zeta U^{2}}{\int_{0}^{1} U^{2}} .
$$

3. It then follows, writing $z_{i}=Z_{i}(0), u(\cdot)=U(0, \cdot)$, that

$$
\begin{aligned}
Z_{i}^{\prime}(0) & =-\frac{V\left(0, z_{i}\right)}{\dot{u}\left(z_{i}\right)}=\frac{\int_{0}^{z_{i}}\left(\zeta-\bar{\zeta}-\Lambda^{\prime}(0)\right) u^{2}}{\dot{u}^{2}\left(z_{i}\right)} \\
& =\frac{1}{\dot{u}^{2}\left(z_{i}\right)}\left\{\int_{0}^{z_{i}} \zeta u^{2}-\frac{\int_{0}^{z_{i}} u^{2}}{\int_{0}^{1} u^{2}} \int_{0}^{1} \zeta u^{2}\right\} \\
& =\frac{1}{\dot{u}^{2}\left(z_{i}\right)} \int_{0}^{1} \zeta u^{2}\left\{\chi_{\left[0, z_{i}\right)}-\frac{\int_{0}^{z_{i}} u^{2}}{\int_{0}^{1} u^{2}}\right\} d x \\
& =\frac{1}{\dot{u}^{2}\left(z_{i}\right)} \int_{0}^{1} \zeta u^{2}\left\{\frac{\int_{z_{i}}^{1} u^{2}}{\int_{0}^{1} u^{2}}-\chi_{\left[z_{i}, 1\right]}\right\} d x .
\end{aligned}
$$


Hence

$$
\left\langle\nabla \mathbf{E}\left(p_{\varepsilon}\right), \zeta-\bar{\zeta}\right\rangle=2 \int_{0}^{1}\left(\dot{p}_{\varepsilon} \dot{\zeta}+\zeta u^{2} \sum_{i=1}^{n-1} \frac{z_{i}-x_{i}}{\varepsilon \dot{u}^{2}\left(z_{i}\right)}\left\{\frac{\int_{z_{i}}^{1} u^{2}}{\int_{0}^{1} u^{2}}-\chi_{\left(z_{i}, 1\right]}\right\}\right) d x .
$$

Thus the first variation of $\mathbf{E}$ at $p_{\varepsilon}$ in any direction $\zeta-\bar{\zeta} \in \mathbf{Q}$ is zero, so that

$$
\dot{p}_{\varepsilon}(0)=\dot{p}_{\varepsilon}(1)=0, \quad \ddot{p}_{\varepsilon}=u^{2} \sum_{i=1}^{n-1} \frac{z_{i}-x_{i}}{\varepsilon \dot{u}^{2}\left(z_{i}\right)}\left\{\frac{\int_{z_{i}}^{1} u^{2}}{\int_{0}^{1} u^{2}}-\chi_{\left(z_{i}, 1\right]}\right\} \quad \text { in }(0,1) .
$$

Set $q_{\varepsilon}=p_{\varepsilon}-\lambda$ and $\mathbf{a}=\left(a_{0}, \cdots, a_{n-1}\right)$, where

$$
\begin{aligned}
& a_{0}=\frac{1}{\varepsilon} \sum_{i=1}^{n-1} \frac{\left(z_{i}-x_{i}\right)}{\dot{u}^{2}\left(z_{i}\right)} \frac{\int_{z_{i}}^{1} u^{2}}{\int_{0}^{1} u^{2}} \\
& a_{k}=a_{0}+\frac{1}{\varepsilon} \sum_{i=1}^{k} \frac{x_{i}-z_{i}}{\dot{u}^{2}\left(z_{i}\right)} \forall k=1, \cdots, n-1 .
\end{aligned}
$$

The minimization problem thus can be formulated as follows:

Problem 4. Given an $\varepsilon>0$, an integer $n \geqslant 1$ and $\mathbf{x}=\left(x_{0}, \cdots, x_{n}\right) \in \mathbf{X}(n)$, find

$$
u, q_{\varepsilon} \in C^{1}([0,1]), \mathbf{a} \in \mathbb{R}^{n}, \mathbf{z} \in \mathbf{X}(n)
$$

such that

$$
\left\{\begin{aligned}
& \ddot{u}= u q_{\varepsilon} \quad \text { in } \quad(0,1), \\
& \dot{u}(0)=1, \quad\{x \in[0,1] \mid u(x)=0\}=\left\{z_{0}, \cdots, z_{n}\right\}, \\
& \ddot{q}_{\varepsilon}= u^{2} \sum_{k=0}^{n-1} a_{k} \chi_{\left[z_{k}, z_{k+1}\right)} \quad \text { in }(0,1), \\
& \dot{q}_{\varepsilon}(0)=\dot{q}_{\varepsilon}(1)=0, \\
& a_{k}= a_{k-1}+\frac{1}{\varepsilon} \frac{x_{k}-z_{k}}{\dot{u}^{2}\left(z_{k}\right)} \quad \forall k=1, \cdots, n, \\
& a_{0} \text { is given by (2.1). }
\end{aligned}\right.
$$

Note that the solvability condition

$$
\begin{aligned}
0 & =\int_{0}^{1} \ddot{q}_{\varepsilon} d x=\sum_{i=0}^{n-1} \int_{z_{i}}^{z_{i+1}} a_{i} u^{2} \sum_{i=0}^{n-1} a_{i}\left(\int_{z_{i}}^{1} u^{2} d x-\int_{z_{i+1}}^{1} u^{2} d x\right) \\
& =\sum_{i=1}^{n-1}\left(a_{i}-a_{i-1}\right) \int_{z_{i}}^{1} u^{2} d x+a_{0} \int_{0}^{1} u^{2} d x
\end{aligned}
$$

and the definition of $a_{i}-a_{i-1}$ in (2.3) automatically give the required formula for $a_{0}$ in (2.1). The inductive definition for $a_{i}$ in (2.3) then also gives the required formula for $a_{i}$ in (2.2) for each $i=1, \cdots, n-1$. Hence, we have proved the following:

Theorem 2.2. Let $\varepsilon>0, n \geqslant 1$, and $\mathbf{x} \in \mathbf{X}(n)$ be given and $p_{\varepsilon}$ be a minimizer to (1.6). Then $\left(\mathbf{z}, \mathbf{a}, u, q_{\varepsilon}\right)$ solves (2.3), where $q_{\varepsilon}=p_{\varepsilon}-\lambda,(\lambda, u)$ is the nth eigenpair to (1.1), $\mathbf{z}$ is the nodal set of $u$, and $\mathbf{a}=\left(a_{0}, \cdots, a_{n-1}\right)$ as in (2.1), (2.2).

Thus, a minimizer can be obtained by first solving (2.3) for $q_{\epsilon}$ and then defining

$$
\lambda:=-\int_{0}^{1} q_{\varepsilon}(x) d x, \quad p_{\varepsilon}=\lambda+q_{\varepsilon} .
$$


In a similar manner, we can obtain the Euler-Lagrange equation for minimizers of $\mathbf{E}_{1}$. Define $\delta a_{i}:=a_{i+1}-a_{i}$. Then

$$
\begin{aligned}
\frac{d}{d t}|\delta \mathbf{x}-\delta Z|^{2} & =2 \sum_{i=1}^{n-1}\left\{\left[Z_{i}-Z_{i-1}\right]-\left[x_{i}-x_{i-1}\right]\right\}\left(Z_{i}^{\prime}-Z_{i-1}^{\prime}\right) \\
& =2 \sum_{i=1}^{n-1}\left\{\left[x_{i+1}-2 x_{i}+x_{i-1}\right]-\left[Z_{i+1}-2 Z_{i}+Z_{i+1}\right]\right\} Z_{i}^{\prime} .
\end{aligned}
$$

Hence, using the notation

$$
\delta^{2} \mathbf{y}_{i}:=y_{i+1}-2 y_{i}+y_{i-1}, \quad i=1, \cdots, n-1, \quad \forall \mathbf{y} \in \mathbf{X}(n), \quad n \geq 2,
$$

we obtain a similar Euler-Lagrange equation for any minimizer of $\mathbf{E}_{1}$ in $\mathbf{Q}$, with the only change that the induction relation among $\left\{a_{i}\right\}_{i=0}^{n-1}$ is replaced by

$$
\delta a_{i}:=\frac{1}{\varepsilon} \frac{\delta^{2} z_{i}-\delta^{2} x_{i}}{\dot{u}\left(z_{i}\right)^{2}}, \quad i=1, \cdots, n-1 .
$$

Similarly, the Euler-Lagrange equation for a minimizer $p_{0}$ of $\mathbf{E}_{2}$ in $\mathbf{Q}^{\mathbf{x}}$ is the following, for an unknown $\left(u, p_{0}, \lambda, \mathbf{a}\right) \in C^{2}([0,1]) \times C^{1}([0,1]) \times \mathbb{R} \times \mathbb{R}^{n}$ :

$$
\left\{\begin{array}{l}
\ddot{u}=\left(p_{0}-\lambda\right) u \quad \text { in }(0,1), \\
\ddot{p}_{0}=u^{2} \sum_{i=0}^{n-1} a_{i} \chi_{\left[x_{i}, x_{i+1}\right)} \quad \text { in }(0,1), \\
\dot{u}(0)=1, \dot{p}_{0}(0)=\dot{p}_{0}(1)=0, \\
\{x \in[0,1] \mid u(x)=0\}=\left\{x_{0}, x_{1}, \cdots, x_{n}\right\} .
\end{array}\right.
$$

Also we remark that (2.3) or (2.4) can be solved numerically by a finite difference method, as shown in Figure 1

Currently, we do not know if the minimizers are unique. Of course, when $n=1$, the minimizers for $\mathbf{E}, \mathbf{E}_{1}$, or $\mathbf{E}_{2}$ are unique, given by $p \equiv 0, \lambda=-\pi^{2}$. Also the system of equation (2.3) appears to be unstable as $\epsilon$ appears in the denominator. However, from the regular numerical solution in the figure, we believe that the system is stable and will leave it to a future study.

\section{3. $L^{r}$ ERROR ESTIMATES FOR SOME RECONSTRUCTION FORMULAS}

Given $\mathbf{x}=\left(x_{0}, \cdots, x_{n}\right) \in \mathbf{X}(n)$, it contains $(n-1)$ independent pieces of information. If we want to recover a potential possessing $\mathbf{x}$ as a nodal set, we can get at most $n-1$ pieces of independent quantified information from $\mathbf{x}$. In this section, we shall show that such information could be the average over each $\left[x_{i}, x_{i+1}\right]$ of the potential. Our motivation is the following observation:

Theorem 3.1. Given an integer $n \geqslant 1$ and $\mathbf{x}=\left(x_{0}, \cdots, x_{n}\right) \in \mathbf{X}(n)$, among all functions which have zero mean and are constants on each $\left(x_{i}, x_{i+1}\right), i=0, \cdots, n-$ 1 , there is one and only one that possesses $\mathbf{x}$ as its nodal set, given by

$$
P(\mathbf{x} ; \cdot):=V(\cdot)-\bar{V}, \quad V(x):=-\sum_{i=0}^{n-1} \frac{\pi^{2} \chi_{\left[x_{i}, x_{i+1}\right)}(x)}{\left(x_{i+1}-x_{i}\right)^{2}}, \quad \bar{V}=\int_{0}^{1} V(x) d x .
$$

The assertion follows by the fact that any eigenfunction on $\left[x_{i}, x_{i+1}\right]$ must be a constant multiple of $\sin \left[\pi\left(x-x_{i}\right) /\left(x_{i+1}-x_{i}\right)\right]$ on $\left[x_{i}, x_{i+1}\right]$. 
We remark that the advantage of $V$ in (3.1) over $2 \pi^{2} n^{3} L_{n}(x)$ in (1.4) is that the former is local; i.e., it does not depend on the total number $n$, which is usually hard to count.

Now suppose $q \in \mathbf{Q}$ is a generic potential that has the nodal set $\mathbf{x}$. We would like to estimate the difference between $q$ and $P(\mathbf{x} ; \cdot)$. Note that a good representative of $q$ is the following piecewise constant mean-value approximation (cf. (1.7)):

$$
\tilde{q}:=\sum_{i=0}^{n-1} \bar{q}_{i} \chi_{\left[x_{i}, x_{i+1}\right)}(x)
$$

In this section, we shall perform two estimates. First we estimate the location of the nodal set $\mathbf{z}(n, q)$ for a given $q \in \mathbf{Q}$ and large enough $n$. Next we estimate the distance between $P(\mathbf{x} ; \cdot)$ and the mean-value approximation $\tilde{q}$ for any $q \in \mathbf{Q}$ satisfying $\mathbf{x}=\mathbf{z}(n, q)$.

Theorem 3.2. Suppose $q \in \mathbf{Q}$ and $n$ is an integer satisfying

$$
\int_{0}^{1}|q| d x \leq \frac{n \pi^{2}}{4}
$$

Let $\lambda=\lambda(n, q)$ be the $n$th eigenvalue and $\mathbf{x}=\mathbf{z}(n, q)$ be the nodal set. Then

$$
\begin{aligned}
& \left|\lambda-(n \pi)^{2}\right| \leqslant \min \left\{\frac{5}{4} \int_{0}^{1}|q| d x, \int_{0}^{1}|q-\tilde{q}| d x+\frac{4 \max _{i}\left\{\overline{|q|}_{i}\right\}+\int_{0}^{1}|q| d x}{(4 n-5 / 4) n \pi^{2}} \int_{0}^{1}|q| d x\right\}, \\
& \left|\left(x_{i}-x_{j}\right)-\frac{i-j}{n}\right| \leqslant \frac{1}{\lambda} \int_{0}^{1}|q| d x<\frac{1}{4 n-5 / 4} \quad \forall i, j, \\
& \left|\bar{q}_{i}-\lambda+\frac{\pi^{2}}{\left(\delta x_{i}\right)^{2}}\right| \leqslant \frac{3}{2 \delta x_{i}} \int_{x_{i}}^{x_{i+1}}\left|q-\bar{q}_{i}\right| d x \quad \forall i
\end{aligned}
$$

Theorem 3.3. Let $\mathbf{x} \in \mathbf{X}(n)$ be given. Define $P, V$ and $\bar{V}$ as in (3.1). Then any $q$ in $\mathbf{Q}$ satisfying $\mathbf{z}(n, q)=\mathbf{x}$ and (3.2) must be close to $P$ in the following sense: For $\lambda=\lambda(n, q), \tilde{q}$ as in (1.7) and every $r \in[1, \infty)$,

$$
|\lambda+\bar{V}| \leqslant\|\lambda+V-\tilde{q}\|_{L^{r}} \leqslant \frac{3}{2}\|q-\tilde{q}\|_{L^{r}}, \quad\|q-P\|_{L^{r}} \leqslant 4\|q-\tilde{q}\|_{L^{r}}
$$

Proof of Theorem 3.2. We divide the proof into the following steps.

1. We use a modified Prüfer substitution:

$$
u(x)=R(x) \sin \theta(x), \quad \dot{u}(x)=m R(x) \cos \theta(x)
$$

where $m>0$ is a constant chosen at our convenience. Then the equations for $u$ give

$$
\left\{\begin{array}{l}
m \dot{\theta}=m^{2}+\left(\lambda-q-m^{2}\right) \sin ^{2} \theta \quad \text { on }[0,1], \\
\theta\left(x_{k}\right)=k \pi \quad \forall k=0, \cdots, n .
\end{array}\right.
$$

We point out that $R(\cdot)>0$ and the solution $\theta(\cdot)$ depends on $m$.

2. Integrating the differential equation in (3.7) over $[0,1]$ and using $\int_{0}^{1} q d x=0$ and $\sin ^{2} \theta=(1-\cos 2 \theta) / 2$, we obtain

$$
\left(\lambda-m^{2}\right) \int_{0}^{1} \sin ^{2} \theta d x=m n \pi-m^{2}-\int_{0}^{1} \frac{q \cos 2 \theta}{2} d x .
$$


Taking $m=n \pi / 2$ gives $\lambda-m^{2}>0$, i.e., $\sqrt{\lambda}>n \pi / 2$. Next setting $m=\sqrt{\lambda}$ we obtain

$$
\begin{aligned}
n \pi & =\sqrt{\lambda}+\frac{1}{2 \sqrt{\lambda}} \int_{0}^{1} q \cos 2 \theta d x \\
\left|(n \pi)^{2}-\lambda\right| & =\left|\left\{1+\frac{1}{4 \lambda} \int_{0}^{1} q \cos 2 \theta d x\right\} \int_{0}^{1} q \cos 2 \theta d x\right| \\
& \leq \frac{5}{4}\left|\int_{0}^{1} q \cos 2 \theta d x\right| \leq \frac{5}{16} n \pi^{2}
\end{aligned}
$$

by using $\lambda>(n \pi)^{2} / 4$ and $\int_{0}^{1}|q| d x \leq n \pi^{2} / 4$.

One can further estimate $\left|\lambda-(n \pi)^{2}\right|$ by using the following:

$$
\begin{aligned}
\int_{0}^{1} q \cos 2 \theta d x & =\sum_{i}\left(\bar{q}_{i} \int_{x_{i}}^{x_{i+1}} \cos 2 \theta d x+\int_{x_{i}}^{x_{i+1}}\left(q-\bar{q}_{i}\right) \cos 2 \theta d x\right) \\
\int_{x_{i}}^{x_{i+1}} \cos 2 \theta d x & =\int_{x_{i}}^{x_{i+1}}\left(\frac{\dot{\theta}}{\sqrt{\lambda}}+\frac{q \sin ^{2} \theta}{\lambda}\right) \cos 2 \theta d x=\frac{1}{\lambda} \int_{x_{i}}^{x_{i+1}} q \sin ^{2} \theta \cos 2 \theta d x .
\end{aligned}
$$

Using $\left|\sin ^{2} \theta \cos 2 \theta\right| \leq 1$, we then obtain

$$
\left|\lambda-(n \pi)^{2}\right| \leq \frac{1}{4 \lambda}\left(\int_{0}^{1}|q| d x\right)^{2}+\frac{\max _{i}\left\{\overline{|q|}_{i}\right\}}{\lambda} \int_{0}^{1}|q| d x+\int_{0}^{1}|q-\tilde{q}| d x .
$$

Finally, using $\lambda>\pi^{2} n\left(n-\frac{5}{16}\right)$ we obtain (3.3).

3. Setting $m=\sqrt{\lambda}$ and integrating (3.7) over $\left[x_{i}, x_{i+1}\right]$ and $[0,1]$ respectively, we obtain

$$
\delta x_{i}=\frac{\pi}{\sqrt{\lambda}}+\frac{1}{\lambda} \int_{x_{i}}^{x_{i+1}} q \sin ^{2} \theta d x, \quad \frac{1}{n}=\frac{\pi}{\sqrt{\lambda}}+\frac{1}{n \lambda} \int_{0}^{1} q \sin ^{2} \theta d x .
$$

Certain linear combinations give

$$
\left|\left(x_{k}-x_{l}\right)-\frac{k-l}{n}\right|=\frac{1}{\lambda}\left|\left(\int_{x_{l}}^{x_{k}}-\frac{k-l}{n} \int_{0}^{1}\right) q \sin ^{2} \theta\right| \leq \frac{1}{\lambda} \int_{0}^{1}|q| d x<\frac{1}{4 n-5 / 4}
$$

for every integer $l<k$ in $[0, n]$. Thus (3.4) follows.

4. For (3.5), we first notice that

$$
\lambda \delta x_{i}=\pi \sqrt{\lambda}+\int_{x_{i}}^{x_{i+1}} q \sin ^{2} \theta d x=\pi \sqrt{\lambda}+\bar{q}_{i} \delta x_{i}-\int_{x_{i}}^{x_{i+1}} q \cos ^{2} \theta d x .
$$

This implies that $\min \left\{\lambda \delta x_{i},\left(\lambda-\bar{q}_{i}\right) \delta x_{i}\right\} \geq \frac{\sqrt{\lambda} \pi}{2}$, so that $\sqrt{\lambda-\bar{q}_{i}} \geq \pi /\left(2 \delta x_{i}\right)$.

Next setting $m=\sqrt{\lambda-\bar{q}_{i}}$ and integrating (3.7) over $\left[x_{i}, x_{i+1}\right]$ we obtain

$$
\sqrt{\lambda-\bar{q}_{i}} \pi=\left(\lambda-\bar{q}_{i}\right) \delta x_{i}+\int_{x_{i}}^{x_{i+1}} \frac{\left(q-\bar{q}_{i}\right) \cos 2 \theta}{2} d x .
$$

Solving this equation for $m=\sqrt{\lambda-\bar{q}_{i}}$ (recalling $\sqrt{\lambda-\bar{q}_{i}} \geq \pi /\left(2 \delta x_{i}\right)$ ), we obtain

$$
\sqrt{\lambda-\bar{q}_{i}}=\frac{\pi}{2 \delta x_{i}}\left\{1+\left(1-\frac{2 \delta x_{i}}{\pi^{2}} \int_{x_{i}}^{x_{i+1}}\left(q-\bar{q}_{i}\right) \cos 2 \theta d x\right)^{1 / 2}\right\} .
$$


The estimate (3.5) then follows by squaring both sides and using

$$
\left|(1+\sqrt{1-s})^{2}-4\right|=\frac{|s|(3+\sqrt{1-s})}{1+\sqrt{1-s}} \leq 3|s| \quad \forall s \in[-1,1] .
$$

This completes the proof of Theorem 3.2

Remark 3.4. The modified Prüfer substitution used here is an extension of the one employed in [1, 10]. In fact, it is equivalent to the transformation

$$
u(x)=R(x) \sin (\sqrt{\lambda} \varphi(x)), \quad \dot{u}(x)=m R(x) \cos (\sqrt{\lambda} \varphi(x)),
$$

where the scaled phase $\varphi:=\theta / \sqrt{\lambda}$ satisfies

$$
\dot{\varphi}=\frac{m}{\sqrt{\lambda}}+\frac{\lambda-q-m^{2}}{m \sqrt{\lambda}} \sin ^{2}(\sqrt{\lambda} \varphi) .
$$

The Prüfer substitution used in [1, 10] indeed corresponds to the choice of $m=\sqrt{\lambda}$, which is usually sufficient for the analysis.

Proof of Theorem 3.3. As the average of $\tilde{q}$ is zero, for any $r \geqslant 1$,

$$
|\lambda+\bar{V}|=\left|\int_{0}^{1}(\lambda+V-\tilde{q}) d x\right| \leqslant\|\lambda+V-\tilde{q}\|_{L^{1}} \leqslant\|\lambda+V-\tilde{q}\|_{L^{r}} .
$$

In addition, using the definition of $V, \tilde{q}$ and (3.5) we have

$$
\begin{aligned}
\|\lambda+V-\tilde{q}\|_{L^{r}}^{r} & =\sum_{i} \delta x_{i}\left|\bar{q}_{i}-\lambda-\frac{\pi^{2}}{\left(\delta x_{i}\right)^{2}}\right|^{r} \leqslant \sum_{i} \delta x_{i}\left(\frac{3}{2 \delta x_{i}} \int_{x_{i}}^{x_{i+1}}\left|q-\bar{q}_{i}\right| d x\right)^{r} \\
& \leqslant\left(\frac{3}{2}\right)^{r} \sum_{i=0}^{n-1} \int_{x_{i}}^{x_{i+1}}\left|q-\bar{q}_{i}\right|^{r} d x=\left(\frac{3}{2}\right)^{r}\|q-\tilde{q}\|^{r} .
\end{aligned}
$$

Thus, $\|\lambda+V-\tilde{q}\|_{L^{r}} \leqslant \frac{3}{2}\|q-\tilde{q}\|_{L^{r}}$. Finally, since $P=V-\bar{V}$,

$$
\|q-P\|_{L^{r}}=\|(q-\tilde{q})+(\tilde{q}-\lambda-V)+(\lambda+\bar{V})\|_{L^{r}} \leq 4\|q-\tilde{q}\|_{L^{r}} .
$$

This completes the proof of Theorem 3.3

Remark 3.5. Since the map from $q$ to $\lambda(n, q)$ is nonlinear, certain conditions such as (3.2) are definitely needed for any linear type estimate such as (3.5) or the first estimate in (3.6) to hold. That means that (3.2) may be relaxed, but cannot be totally removed. Consider the following example. Fix any constant $A>0$, define $h=\arctan (\operatorname{coth} A), \Lambda=2\left(h^{2}-A^{2}\right)$, and

$$
\begin{aligned}
& U(y)= \begin{cases}\frac{\sinh (2[A+h] y)}{\sinh A} & \text { if } y \in\left[0, \frac{A}{2(A+h)}\right], \\
\frac{\cos ([A+h][1-2 y])}{\cos h} & \text { if } y \in\left[\frac{A}{2(A+h)}, \frac{1}{2}\right],\end{cases} \\
& Q(y)= \begin{cases}4 h[A+h] & \text { if } y \in\left[0, \frac{A}{2(A+h)}\right], \\
-4 A[A+h] & \text { if } y \in\left[\frac{A}{2(A+h)}, \frac{1}{2}\right],\end{cases} \\
& U(y)=-U(-y)=U(1-y), \quad Q(-y)=Q(y)=Q(1-y), \quad \forall y \in \mathbb{R} .
\end{aligned}
$$

Note that $h \rightarrow \pi / 4$ and $\Lambda \rightarrow-\infty$ as $A \rightarrow \infty$. Also

$$
U_{y y}=(Q-\Lambda) U \quad \text { in } \mathbb{R}, \quad\{y \mid U(y)=0\}=\mathbb{Z} .
$$

Fix any integer $n \geq 1$ and set $\mathbf{x}=\left(0, \frac{1}{n}, \frac{2}{n}, \cdots, \frac{n-1}{n}, 1\right) \in \mathbf{X}(n), \quad q(x)=n^{2} Q(n x), \quad u(x)=U(n x), \quad \lambda=n^{2} \Lambda$. 
The family $\{q\}_{A>1}$ has the property that $\mathbf{x}=\mathbf{z}(n, q)$, but

$$
\lim _{A \rightarrow \infty} \frac{\lambda(n, q)}{\int_{0}^{1}|q| d x}=\lim _{A \rightarrow \infty} \frac{2\left(h^{2}-A^{2}\right)}{8 A h}=-\infty \quad \forall n=1,2, \cdots .
$$

Since $\tilde{q} \equiv 0$, the inequality (3.5) does not hold for all $A>1$; even the constant $\frac{3}{2}$ is replaced by any larger constant.

For the second estimate in (3.6), we do not know if it holds unconditionally when the constant " 4 " is replaced by a certain large number.

Remark 3.6. As a demonstration, we provide an estimate for the $L^{r}=L^{r}((0,1))$ difference between $q$ and $\tilde{q}$ :

$$
\begin{aligned}
\|q-\tilde{q}\|_{L^{r}}^{r} & :=\int_{0}^{1}|q-\tilde{q}|^{r} d x=\sum_{i} \int_{x_{i}}^{x_{i+1}}\left|\frac{1}{\delta x_{i}} \int_{x_{i}}^{x_{i+1}}(q(x)-q(y)) d y\right|^{r} d x \\
& \leq \sum_{i} \frac{1}{\delta x_{i}} \int_{x_{i}}^{x_{i+1}} d x \int_{x_{i}}^{x_{i+1}}|q(x)-q(y)|^{r} d y .
\end{aligned}
$$

Set

$$
\delta^{*} \mathbf{x}=\max \left\{\delta x_{0}, \cdots, \delta x_{n-1}\right\}, \quad \delta_{*} \mathbf{x}=\min \left\{\delta x_{0}, \cdots, \delta x_{n-1}\right\} .
$$

By (3.4), we have

$$
\frac{2}{3 n}<\delta_{*} \mathbf{x}<\delta^{*} \mathbf{x}<\frac{4}{3 n} .
$$

For any exponent $r \in[1, \infty)$ we have

$$
\begin{aligned}
\|q-\tilde{q}\|_{L^{r}}^{r} & \leq \sum_{i} \frac{1}{\delta x_{i}} \int_{x_{i}}^{x_{i+1}} \int_{x_{i}}^{x_{i+1}}|q(t)-q(s)|^{r} d t d s \\
& \leq \frac{1}{\delta_{*} \mathbf{x}} \int_{0}^{1} \int_{\max \left\{-\delta^{*} \mathbf{x},-t\right\}}^{\min \left\{\delta^{*} \mathbf{x}, 1-t\right\}}|q(t)-q(t+h)|^{r} d h d t \\
& =\frac{1}{\delta_{*} \mathbf{x}} \int_{-\delta^{*} \mathbf{x}}^{\delta^{*} \mathbf{x}} \int_{\min \{-h,-x\}}^{\min \{1,1-h\}}|q(t)-q(t+h)|^{r} d t d h \\
& \leq \frac{2 \delta^{*} \mathbf{x}}{\delta_{*} \mathbf{x}} \sup _{0<h<\delta x^{*}} \int_{0}^{1-h}|f(t)-f(t+h)| d t \\
& \leq 4 \sup _{0<h<4 /(3 n)} \int_{0}^{1-h}|q(t)-q(t+h)|^{r} d t .
\end{aligned}
$$

Proof of Theorem 1.1. The first part was proved in Theorem 3.3 . It remains to show the second part. For this, let $k_{0}, \cdots, k_{J}$ be integers satisfying $0=k_{0}<k_{1}<$ $\cdots<k_{J}=n$. Let $L_{n}$ be defined by

$$
\tilde{L}_{n}(x)=\sum_{j=1}^{J} \frac{x_{k_{j}}^{(n)}-x_{k_{j-1}}^{(n)}}{k_{j}-k_{j-1}} \chi_{\left[x_{k_{j}-1}^{(n)}, x_{k_{j}}^{(n)}\right)}(x) .
$$

We shall estimate the $L^{r}$ difference between $2 n^{2} \pi^{2}\left(n \tilde{L}_{n}-1\right)$ and $q$, where $\mathbf{x}=$ $\mathbf{z}(n, q)$.

Let $l, k$ be any integers satisfying $0 \leqslant l<k \leqslant n$. Setting $m=n \pi$ and integrating $1=\frac{\dot{\theta}}{n \pi}+\frac{q+n^{2} \pi^{2}-\lambda}{n^{2} \pi^{2}} \sin ^{2} \theta$ over $\left[x_{l}, x_{k}\right]$, we obtain

$$
x_{k}-x_{l}=\frac{k-l}{n}+\frac{1}{n^{2} \pi^{2}} \int_{x_{l}}^{x_{k}}\left(q+n^{2} \pi^{2}-\lambda\right) \sin ^{2} \theta d x .
$$


Denote

$$
\begin{gathered}
\ell:=\frac{n\left(x_{k}-x_{l}\right)}{k-l}, \quad f\{\} d x:=\frac{1}{x_{k}-x_{l}} \int_{x_{l}}^{x_{k}}\{\} d x \\
W:=f\left(q+n^{2} \pi^{2}-\lambda\right) \sin ^{2} \theta d x . \quad \bar{q}:=f q d x .
\end{gathered}
$$

We then obtain the relation

$$
\ell-1=\frac{\ell}{n^{2} \pi^{2}} W, \quad \ell=\frac{1}{1-W /\left(n^{2} \pi^{2}\right)} .
$$

Note that (3.4) implies that

$$
|\ell-1| \leq \frac{n}{(k-l)(4 n-5 / 4)} \leq \frac{1}{3} \quad \Rightarrow \quad \frac{|W|}{n^{2} \pi^{2}} \leq \frac{1}{2} .
$$

The previous relation can be expressed in the form

$$
2 n^{2} \pi^{2}(\ell-1)-\bar{q}=2 \ell W-\bar{q}
$$

Using the definition of $W$ we have

$$
\begin{aligned}
2 W= & 2 f(q-\bar{q}) \sin ^{2} \theta d x \\
& +2\left(\bar{q}+n^{2} \pi^{2}-\lambda\right) f \sin ^{2} \theta\left\{\frac{d \theta}{n \pi}+\frac{\left(q+n^{2} \pi^{2}-\lambda\right) \sin ^{2} \theta d x}{n^{2} \pi^{2}}\right\} \\
= & \frac{\bar{q}+n^{2} \pi^{2}-\lambda}{\ell}+f(\bar{q}-q) \cos (2 \theta) d x \\
& +\frac{2\left(\bar{q}+n^{2} \pi^{2}-\lambda\right)}{n^{2} \pi^{2}} f\left(q+n^{2} \pi^{2}-\lambda\right) \sin ^{4} \theta d x .
\end{aligned}
$$

Upon using the estimate $\left|\lambda-n^{2} \pi^{2}\right| \leq \frac{5}{4} \int_{0}^{1}|q|$ and $|\ell| \leq \frac{4}{3}$, we then obtain

$$
|2 \ell W-\bar{q}| \leq\left|\lambda-n^{2} \pi^{2}\right|+\frac{4}{3} f|q-\bar{q}|+\frac{8}{3 n^{2} \pi^{2}}\left(\overline{|q|}+\frac{5}{4} \int_{0}^{1}|q| d x\right)^{2} .
$$

Now take $l=k_{j-1}, k=k_{j}$. Denote the corresponding $\ell, W, \bar{q}$ by $\ell_{j}, W_{j}, \bar{q}_{j}$, and set

$$
\hat{\ell}:=\sum_{j=1}^{J} \ell_{j} \chi_{\left[x_{k_{j-1}}, x_{k_{j}}\right)}(x), \quad \hat{W}:=\sum_{j=1}^{J} W_{j} \chi_{\left[x_{k_{j-1},}, x_{k_{j}}\right)}(x) .
$$

Then, by (3.3),

$$
\begin{aligned}
\left|\lambda-n^{2} \pi^{2}\right| & \leq \frac{5}{4} \int_{0}^{1}|q| d x \leq \frac{5}{4} \max \overline{|q|}_{j} \\
\left|\lambda-n^{2} \pi^{2}\right| & \leq \frac{\left.5 \max _{j} \overline{|q|}\right|_{j}}{n \pi^{2}(4 n-5 / 4)} \int_{0}^{1}|q| d x+\int_{0}^{1}|q-\tilde{q}| \leq \frac{2 \max _{j} \overline{|q|}_{j}}{n^{2} \pi^{2}}\|q\|_{1}+\|q-\tilde{q}\|_{1} .
\end{aligned}
$$


Then we have, for each $r \geq 1$, from (3.12),

$$
\begin{aligned}
& \left\|2 n^{2} \pi^{2}[n \tilde{L}(x)-1]-\tilde{q}\right\|_{r} \\
& =\|2 \hat{\ell} \hat{W}-\tilde{q}\|_{r} \\
& \leq\left|\lambda-n^{2} \pi^{2}\right|+\frac{4}{3}\left\|\sum_{j=1}^{J} \frac{\chi_{\left[x_{k_{j-1}, x_{k_{j}}}\right)}(x)}{\delta x_{k_{j-1}}} \int_{x_{k_{j-1}}}^{x_{k_{j}}}\left|q-\bar{q}_{j}\right|\right\|_{r} \\
& \quad \quad+\frac{8}{3 n^{2} \pi^{2}}\left\|\left(\overline{|q|}+\frac{5}{4} \int_{0}^{1}|q| d x\right)^{2}\right\|_{r} \\
& \leq \frac{2 \max _{j} \overline{|q|}_{j}}{n^{2} \pi^{2}}\|q\|_{1}+\|q-\tilde{q}\|_{1}+\frac{4}{3}\|q-\tilde{q}\|_{r}+\frac{8}{3 n^{2} \pi^{2}}\left(\frac{81}{16} \max _{j} \overline{|q|}_{j}\|q\|_{r}\right) \\
& \leq \frac{7}{3}\|q-\tilde{q}\|_{r}+\frac{31 \max _{j} \overline{|q|}_{j}}{2 n^{2} \pi^{2}}\|q\|_{r} .
\end{aligned}
$$

In the next to last line above, the following estimate is used. This estimate is itself a consequence of Hölder's inequality.

$$
\begin{aligned}
\left\|\overline{q q}^{2}\right\|_{r} & =\left[\sum_{j=1}^{J}\left(\frac{1}{\delta x_{k_{j-1}}} \int_{x_{k_{j-1}}}^{x_{k_{j}}}|q|\right)^{2 r} \delta x_{k_{j-1}}\right]^{\frac{1}{r}} \\
& \leq \max _{j} \overline{|q|}_{j}\left[\sum_{j=1}^{J}\left(\frac{1}{\delta x_{k_{j-1}}} \int_{x_{k_{j-1}}}^{x_{k_{j}}}|q|\right)^{r} \delta x_{k_{j-1}}\right]^{\frac{1}{r}} \\
& \leq \max _{j} \overline{|q|}_{j}\left[\sum_{j=1}^{J} \int_{x_{k_{j-1}}}^{x_{k_{j}}}|q|^{r}\right]^{\frac{1}{r}} \\
& =\max _{j} \overline{|q|}_{j}\|q\|_{r} .
\end{aligned}
$$

This completes the proof.

We remark that the quadratic term cannot be removed because when $q$ is piecewise constant, $2 \pi^{2} n^{2}\left(n \tilde{L}_{n}-1\right)$ is not the exact solution.

\section{Error estimates for Tikhonov Regularization method}

We first study a variation of a potential with respect to its nodal set; that is, we want to estimate the difference $q_{1}-q_{2}$ in terms of the difference $\mathbf{z}\left(n, q_{1}\right)-\mathbf{z}\left(n, q_{2}\right)$. Then we shall use it to prove Theorem 2. For definiteness, we shall use the $L^{2}$ space for potentials and the Euclidean distance for nodal sets (points in $\mathbb{R}^{n+1}$ ). Since it is impossible to determine completely a potential from a single given nodal set, we supply the rest of the information by an a priori bound on the $H^{1}$ norms of the potential.

Given $q_{1}, q_{2} \in \mathbf{Q}$ and $n$ a positive integer such that

$$
\int_{0}^{1}\left|q_{1}\right| d x \leq \frac{n \pi^{2}}{4}, \quad \int_{0}^{1}\left|q_{2}\right| d x \leq \frac{n \pi^{2}}{4},
$$


set $\mathbf{x}:=\mathbf{z}\left(n, q_{1}\right), \mathbf{y}:=\mathbf{z}\left(n, q_{2}\right)$ and

$$
P_{i}=V_{i}-\bar{V}_{i}, \quad \text { where } V_{1}=-\sum_{i=0}^{n-1} \frac{\pi^{2} \chi_{\left[x_{i}, x_{i+1}\right)}}{\left(\delta x_{i}\right)^{2}}, \quad V_{2}=-\sum_{n=0}^{n-1} \frac{\pi^{2} \chi_{\left[y_{i}, y_{i+1}\right)}}{\left(\delta y_{i}\right)^{2}}
$$

Set $|\delta(\mathbf{x}-\mathbf{y})|^{2}:=\sum_{i}\left|\delta x_{i}-\delta y_{i}\right|^{2}=\sum_{i}\left|\left(x_{i+1}-x_{i}\right)-\left(y_{i+1}-y_{i}\right)\right|^{2}$. Then we have

Theorem 4.1. There is a universal constant $C>0$ such that

$$
\begin{aligned}
\int_{0}^{1}\left|q_{1}-q_{2}\right|^{2} & \leq C\left\{n^{5}|\delta(\mathbf{x}-\mathbf{y})|^{2}+\frac{1}{n^{2}} \int_{0}^{1}\left(\dot{q}_{1}^{2}+\dot{q}_{2}^{2}\right) d x\right\} \\
\left\|V_{1}-V_{2}\right\|_{L^{2}(0,1)}^{2} & \leq C\left\{n^{5}|\delta(\mathbf{x}-\mathbf{y})|^{2}+\frac{1}{n^{2}} \int_{0}^{1}\left(\dot{q}_{1}^{2}+\dot{q}_{2}^{2}\right) d x\right\}, \\
n^{5}|\delta(\mathbf{x}-\mathbf{y})|^{2} & \leq C\left\{\int_{0}^{1}\left|q_{1}-q_{2}\right|^{2} d x+\frac{1}{n^{2}} \int_{0}^{1}\left(\dot{q}_{1}^{2}+\dot{q}_{2}^{2}\right) d x\right\} .
\end{aligned}
$$

Proof. First, (4.1) follows from (3.6) and (4.2). Indeed, writing $\|\cdot\|_{L^{2}}$ as $\|\cdot\|$,

$$
\left\|q_{1}-q_{2}\right\| \leq\left\|q_{1}-\tilde{q}_{1}\right\|+\left\|q_{2}-\tilde{q}_{2}\right\|+\left\|\tilde{q}_{2}-P_{2}\right\|+\left\|\tilde{q}_{1}-P_{1}\right\|+\left\|P_{1}-P_{2}\right\| .
$$

The term $\left\|P_{1}-P_{2}\right\|$ can be bounded by $\left\|V_{1}-V_{2}\right\|$, whereas the term $\left\|q_{i}-\tilde{q}_{i}\right\|$ can be bounded by $\left\|\dot{q}_{i}\right\|_{L^{2}}$, using the following Poincaré inequality:

$$
\int_{a}^{b}|q-\bar{q}|^{2} d x \leq \frac{4(b-a)^{2}}{\pi^{2}} \int_{a}^{b} \dot{q}^{2}(x) d x \quad \forall q \in H^{1}((a, b)), a<b .
$$

For (4.2), we use the fact that $\max _{i}\left\{\left|x_{i}-i / n\right|,\left|y_{i}-i / n\right|\right\} \leq 1 /(4 n-5 / 4)$ which implies $\left[x_{i}, x_{i+1}\right] \subset\left[y_{i-1}, y_{i+2}\right]$ for each $i$ (here for simplicity $y_{-1}:=-1 / n, y_{n+1}:=$ $1+1 / n)$. Denote

$$
\begin{aligned}
& \delta_{i}^{+}=\max \left\{0, y_{i}-x_{i}\right\}, \quad \delta_{i}^{-}=\max \left\{0, x_{i}-y_{i}\right\}, \quad \delta_{i}=\left|x_{i}-y_{i}\right| \\
& \delta^{*}=\max \left\{\delta^{*} \mathbf{x}, \delta^{*} \mathbf{y}\right\}=\max _{i}\left\{\delta x_{i}, \delta y_{i}\right\} \\
& \delta_{*}=\min \left\{\delta_{*} \mathbf{x}, \delta_{*} \mathbf{y}\right\}=\min _{i}\left\{\delta x_{i}, \delta y_{i}\right\}
\end{aligned}
$$

Then

$$
\begin{aligned}
& \frac{1}{\pi^{4}}\left\|V_{1}-V_{2}\right\|_{L^{2}}^{2}=\sum_{i} \int_{x_{i}}^{x_{i+1}}\left|\frac{1}{\delta x_{i}^{2}}-\sum_{j=i-1}^{i+1} \frac{\chi_{\left[y_{j}, y_{j+1}\right)}}{\delta y_{j}^{2}}\right|^{2} d x \\
= & \sum_{i}\left|\frac{1}{\delta x_{i}^{2}}-\frac{1}{\delta y_{i}^{2}}\right|^{2}\left(\delta x_{i}-\delta_{i}^{+}-\delta_{i+1}^{-}\right) \\
& +\sum_{i}\left|\frac{1}{\delta x_{i}^{2}}-\frac{1}{\delta y_{i-1}^{2}}\right|^{2} \delta_{i}^{+}+\sum_{i}\left|\frac{1}{\delta y_{i+1}^{2}}-\frac{1}{\delta x_{i}^{2}}\right|^{2} \delta_{i+1}^{-} \\
\leq & \sum_{i}\left|\frac{1}{\delta x_{i}^{2}}-\frac{1}{\delta y_{i}^{2}}\right|^{2}\left(\delta x_{i}+\delta_{i}^{+}+\delta_{i+1}^{-}\right) \\
& +2 \sum_{i}\left|\frac{1}{\delta y_{i}^{2}}-\frac{1}{\delta y_{i-1}^{2}}\right|^{2} \delta_{i}^{+}+2 \sum_{i}\left|\frac{1}{\delta y_{i+1}^{2}}-\frac{1}{\delta y_{i}^{2}}\right|^{2} \delta_{i+1}^{-} \\
\leq & 2 \sum_{i}\left|\frac{1}{\delta x_{i}^{2}}-\frac{1}{\delta y_{i}^{2}}\right|^{2} \delta x_{i}+2 \sum_{i}\left|\frac{1}{\delta y_{i-1}^{2}}-\frac{1}{\delta y_{i}^{2}}\right|^{2} \delta_{i}
\end{aligned}
$$

by using $(a+b)^{2} \leq 2\left(a^{2}+b^{2}\right)$. The first part can be easily estimated by

$$
\sum_{i}\left|\frac{1}{\delta x_{i}^{2}}-\frac{1}{\delta y_{i}^{2}}\right|^{2} \delta x_{i} \leq \frac{4}{\delta_{*}^{5}} \sum_{i}\left|\delta x_{i}-\delta y_{i}\right|^{2} .
$$


To estimate the second term we use the Sturm comparison theorem on zeros to obtain

$$
\min _{\left[y_{i}, y_{i+1}\right]}\left(\lambda\left(n, q_{2}\right)-q_{2}\right) \leq \frac{\pi^{2}}{\delta y_{i}^{2}} \leq \max _{\left[y_{i}, y_{i+1}\right]}\left(\lambda\left(n, q_{2}\right)-q_{2}\right) .
$$

Thus

$$
\begin{aligned}
\left|\frac{\pi^{2}}{\delta y_{i-1}^{2}}-\frac{\pi^{2}}{\delta y_{i}^{2}}\right|^{2} & \leq \mid \max _{\left[y_{i-1}, y_{i+1}\right]} q_{2}-\underset{\left[y_{i-1}, y_{i+1}\right]}{\min _{2}} q_{2}^{2} \\
& \leq\left(\int_{y_{i-1}}^{y_{i+1}}\left|\dot{q}_{2}\right| d y\right)^{2} \leq\left(y_{i+1}-y_{i-1}\right) \int_{y_{i-1}}^{y_{i+1}} \dot{q}_{2}^{2} d y .
\end{aligned}
$$

Therefore, $\delta_{i} \leq \delta^{*}$, and by (3.10),

$$
\begin{aligned}
\left\|V_{1}-V_{2}\right\|_{L^{2}(0,1)}^{2} & \leq \frac{8 \pi^{4}}{\delta_{*}^{5}}|\delta(\mathbf{x}-\mathbf{y})|^{2}+8 \delta^{* 2} \int_{0}^{1}\left|\dot{q}_{2}\right|^{2} d y \\
& \leq \frac{(3 n)^{5}}{4}|\delta(\mathbf{x}-\mathbf{y})|^{2}+\frac{128}{9 n^{2}} \int_{0}^{1}\left|\dot{q}_{2}\right|^{2} d y .
\end{aligned}
$$

This proves (4.2).

The proof of (4.3) follows from the observation that

$$
\frac{4}{\left(\delta^{*}\right)^{5}}\|\delta(\mathbf{x}-\mathbf{y})\|^{2} \leq \sum\left|\frac{1}{\delta x_{i}^{2}}-\frac{1}{\delta y_{i}^{2}}\right|^{2} \delta x_{i} \leq\left\|V_{1}-V_{2}\right\|^{2} \max _{i} \frac{\delta x_{i}}{\delta x_{i}-\delta_{i}^{+}-\delta_{i+1}^{-}}
$$

Also,

$$
\begin{aligned}
\left\|V_{1}-V_{2}\right\| \leq\left\|q_{1}-q_{2}\right\|+\left|\lambda_{1}-(n \pi)^{2}\right|+\left|\lambda_{2}-(n \pi)^{2}\right| \\
+\left\|\lambda_{1}+V_{1}-\tilde{q}_{1}\right\|+\left\|\lambda_{2}+V_{2}-\tilde{q}_{2}\right\|+\left\|q_{1}-\tilde{q}_{1}\right\|+\left\|q_{2}-\tilde{q}_{2}\right\| .
\end{aligned}
$$

By Theorems 3.2 and 3.3 , each term on the right-hand side except the first term can be bounded by $\left\|\dot{q}_{1}\right\|+\left\|\dot{q}_{2}\right\|$.

Proof of Theorem 1.2 . Recall that $\left\|\dot{p}_{\varepsilon}\right\| \leq\|\dot{q}\|$. Then notice that, since the average of $q$ is zero,

$$
\int_{0}^{1}|q| d x \leq\left(\int_{0}^{1} q^{2} d x\right)^{1 / 2} \leq\left(\frac{4}{\pi^{2}} \int_{0}^{1} \dot{q}^{2} d x\right)^{1 / 2} \leq \frac{n \pi^{2}}{4} .
$$

The same estimates also hold for $p_{\varepsilon}$. Note that $\|\delta(\mathbf{x}-\mathbf{y})\|^{2} \leq 2|\mathbf{x}-\mathbf{y}|^{2}$. Thus, we can apply (4.1) to conclude that

$$
\begin{aligned}
\int_{0}^{1}\left|p_{\varepsilon}-q\right|^{2} d x & \leq C n^{5}\left|\mathbf{z}\left(n, p_{\varepsilon}\right)-\mathbf{x}\right|^{2}+\frac{C}{n^{2}} \int_{0}^{1}\left(\dot{p}_{\varepsilon}^{2}+\dot{q}^{2}\right) d x \\
& =C n^{5}\left(\varepsilon \mathbf{E}\left(\mathbf{x}, p_{\varepsilon}\right)-\varepsilon \int_{0}^{1} \dot{p}_{\varepsilon}^{2}\right)+\frac{C}{n^{2}} \int_{0}^{1}\left(\dot{p}_{\varepsilon}^{2}+\dot{q}^{2}\right) d x \\
& \leq \frac{C}{n^{2}}\left\{\left(n^{7} \varepsilon+1\right) \int_{0}^{1} \dot{q}^{2} d x+\left(1-n^{7} \varepsilon\right) \int_{0}^{1} \dot{p}_{\varepsilon}^{2} d x\right\} \\
& \leq C\left(\frac{1}{n^{2}}+\varepsilon n^{5}\right) \int_{0}^{1} \dot{q}^{2} d x
\end{aligned}
$$

On the other hand,

$$
\begin{aligned}
\left\|p_{\varepsilon}^{\alpha}-q\right\|^{2} & \leq 2\left\|p_{\varepsilon}^{\alpha}-q^{\alpha}\right\|^{2}+2\left\|q^{\alpha}-q\right\|^{2} \\
& \leq C\left(\frac{1}{n^{2}}+\varepsilon n^{5}\right)\left\|\dot{q}^{\alpha}\right\|^{2}+2\left\|q^{\alpha}-q\right\|^{2}
\end{aligned}
$$


where

$$
\begin{aligned}
\left\|q^{\alpha}-q\right\|^{2} & \leq C\left(n^{5}\left|\mathbf{z}\left(n, q^{\alpha}\right)-\mathbf{x}\right|^{2}+\frac{1}{n^{2}}\left(\left\|\dot{q}^{\alpha}\right\|^{2}+\|\dot{q}\|^{2}\right)\right) \\
& \leq C\left(n^{5} \alpha^{2}+\frac{1}{n^{2}}\left(\left\|\dot{q}^{\alpha}\right\|^{2}+\|\dot{q}\|^{2}\right)\right) .
\end{aligned}
$$

Therefore,

$$
\left\|p_{\varepsilon}^{\alpha}-q\right\|^{2} \leq 2 C n^{5} \alpha^{2}+\frac{2 C}{n^{2}}\|\dot{q}\|^{2}+C\left(\frac{3}{n^{2}}+\varepsilon n^{5}\right)\left\|\dot{q}^{\alpha}\right\|^{2} .
$$

\section{Appendix}

Here we derive the asymptotic expansion (1.2). Consider the equation $\ddot{u}+V u=$ 0 , where $V$ is positive and large. Suppose $\mathbf{x}=\left(x_{0}, x_{1}, \cdots, x_{n}\right)$ is the nodal set of $u$. The modified Prüfer transformation $u=R(x) \sin \theta(x), \dot{u}=m R(x) \sin \theta(x)$, where $m$ is a large positive constant to be chosen at our convenience, gives the equivalent form

$$
1=\frac{\dot{\theta}}{m}+\frac{p(x) \sin ^{2} \theta}{m^{2}}, \quad \theta\left(x_{k}\right)=k \pi \quad \forall k, \quad p(x):=m^{2}-V(x) .
$$

In each interval $I_{k}=\left[x_{k}, x_{k+1}\right]$, we shall take the constant $m$ to be close to $\sqrt{V\left(x_{k}\right)}$, so $m$ is a large constant. Then $p$ is not very large in $I_{k}$, so the equation can be regarded as a small perturbation from the equation $\theta^{\prime}=m$. We shall first derive an expansion for a general $m$, and then take a particular $m$ to make the leading error term vanish.

Integrating the equation over $I_{k}:=\left[x_{k}, x_{k+1}\right]$ and using $d x=\frac{d \theta}{m}+\frac{p \sin ^{2} \theta d x}{m^{2}}$ we obtain

$$
\begin{aligned}
x_{k+1} & -x_{k}-\frac{\pi}{m}=\int_{I_{k}} \frac{p \sin ^{2} \theta}{m^{2}} d x=\int_{I_{k}} \frac{p \sin ^{2} \theta}{m^{2}}\left\{\frac{d \theta}{m}+\frac{p \sin ^{2} \theta d x}{m^{2}}\right\} \\
= & \left.\frac{[2 \theta-(2 k+1) \pi-\sin (2 \theta)] p}{4 m^{3}}\right|_{x_{k}} ^{x_{k+1}}+\int_{I_{k}} \frac{p^{2} \sin ^{4} \theta}{m^{4}} d x \\
& +\int_{I_{k}} \frac{[(2 k+1) \pi-2 \theta+\sin (2 \theta)] \dot{p}}{4 m^{3}}\left\{\frac{d \theta}{m}+\frac{p \sin ^{2} \theta d x}{m^{2}}\right\} \\
= & \frac{\left[p\left(x_{k}\right)+p\left(x_{k+1}\right] \pi\right.}{4 m^{3}}+\frac{1}{m^{4}} \int_{I_{k}}\left\{p^{2} \sin ^{4} \theta-\frac{1}{4}\left[(\theta-k \pi)([k+1] \pi-\theta)+\sin ^{2} \theta\right] \ddot{p}\right\} d x \\
& -\frac{1}{m^{5}} \int_{I_{k}}[2 \theta-(2 k+1) \pi-\sin (2 \theta)] \dot{p} p \sin ^{2} \theta d x \\
= & \frac{\left[p\left(x_{k}\right)+p\left(x_{k+1}\right] \pi\right.}{4 m^{3}}+\frac{p^{2}\left(\frac{1}{2}\left[x_{k}+x_{k+1}\right]\right)}{m^{5}} \int_{0}^{\pi} \sin ^{4} \theta d \theta \\
& -\frac{\ddot{p}\left(\frac{1}{2}\left[x_{k}+x_{k+1}\right]\right)}{4 m^{5}} \int_{0}^{\pi}\left\{(\theta-k \pi)([k+1] \pi-\theta)+\sin ^{2} \theta\right\} d \theta+\frac{O(1)}{m^{6}} \\
= & \frac{\left[p\left(x_{k}\right)+p\left(x_{k+1}\right)\right] \pi}{4 m^{3}}+\frac{3 \pi}{8 m^{5}}\left\{p^{2}\left(\frac{1}{2}\left[x_{k}+x_{k+1}\right]\right)-\frac{3+\pi^{2}}{9} \ddot{p}\left(\frac{1}{2}\left[x_{k}+x_{k+1}\right]\right)\right\}+\frac{O(1)}{m^{6}} .
\end{aligned}
$$

Here $O(1)$ depends on the bounds of $p:=m^{2}-V$ and its derivatives. 
1. Now we take $m=m_{k}$ such that $p\left(x_{k}\right)+p\left(x_{k+1}\right)=0$. This yields

$$
x_{k}-x_{k-1}=\frac{\pi}{m_{k}}+\frac{O(1)}{m_{k}^{5}}, \quad m_{k}:=\sqrt{\frac{V\left(x_{k}\right)+V\left(x_{k+1}\right)}{2}} .
$$

We remark that if one takes other choices of $m^{2}$, say $m^{2}=V\left(\frac{1}{2}\left[x_{k}+x_{k+1}\right]\right)$ or the average of $V$ over $\left[x_{k}, x_{k+1}\right]$, then the remainder term in general has only the order of $O(1) m^{-4}$.

Then the previous expansion can be written as

$$
\sqrt{\frac{2 \pi^{2}}{V\left(x_{k}\right)+V\left(x_{k+1}\right)}}=\left(x_{k+1}-x_{k}\right)\left\{1+O(1)\left(x_{k+1}-x_{k}\right)^{4}\right\} .
$$

This provides a reconstruction formula

$$
\frac{V\left(x_{k}\right)+V\left(x_{k+1}\right)}{2}=\left(\frac{\pi}{x_{k+1}-x_{k}}\right)^{2}\left\{1+O(1)\left(x_{k+1}-x_{k}\right)^{4}\right\} \quad \forall k .
$$

2. Suppose $V=\lambda-q$, where $\lambda$ is a large constant and $q$ is a smooth and bounded function. Then the above gives the expansion

$$
x_{k+1}-x_{k}=\frac{\pi}{\sqrt{\lambda}}\left\{1+\frac{q_{k}+q_{k+1}}{4 \lambda}+\frac{O(1)}{\lambda^{2}}\right\} .
$$

Assume further that $x_{n}-x_{0}=1$ and $\int_{x_{0}}^{x_{n}} q d x=0$. Taking the sum of the above expansions from $k=0$ to $n-1$ we then obtain successively the following information:

$$
\begin{aligned}
1=\frac{n \pi}{\sqrt{\lambda}}\{1+o(1)\}, & 1=\frac{n \pi}{\sqrt{\lambda}}\left\{1+\frac{O(1)}{n^{2}}\right\}, \quad x_{k}-x_{k-1}=\frac{1}{n}+\frac{O(1)}{n^{3}}, \\
\sum_{k=0}^{n-1} \frac{q\left(x_{k}\right)+q\left(x_{k+1}\right)}{2 n} & =\sum_{k=0}^{n-1}\left(x_{k+1}-x_{k}\right) \frac{q\left(x_{k}\right)+q\left(x_{k+1}\right)}{2}+\sum_{k=0}^{n-1} \frac{O(1)}{n^{3}} \\
& =\int_{x_{0}}^{x_{n}} q(x) d x+\sum_{k=0}^{n-1} O\left(\left[x_{k+1}-x_{k}\right]^{3}\right)+\frac{O(1)}{n^{2}}=\frac{O(1)}{n^{2}} .
\end{aligned}
$$

It then follows that

$$
1=\frac{n \pi}{\sqrt{\lambda}}\left\{1+\frac{O(1)}{n^{4}}\right\}, \quad \sqrt{\lambda}=n \pi\left\{1+\frac{O(1)}{n^{4}}\right\} .
$$

Consequently, we have the following asymptotic expansions:

$$
\begin{aligned}
x_{k+1}-x_{k} & =\frac{1}{n}+\frac{q\left(x_{k}\right)+q\left(x_{k+1}\right)}{2 n^{3} \pi^{2}}+\frac{O(1)}{n^{5}} \\
& =\frac{1}{n}+\frac{q\left(\frac{1}{2}\left[x_{k}+x_{k+1}\right]\right)}{4 n^{3} \pi^{2}}+\frac{O(1)}{n^{5}} \\
& =\frac{1}{n}+\frac{1}{2 n^{3} \pi^{2}}\left(\frac{1}{x_{k+1}-x_{k}} \int_{x_{k}}^{x_{k+1}} q(x) d x\right)+\frac{O(1)}{n^{5}} .
\end{aligned}
$$

\section{ACKNOWLEDGEMENT}

The first author thanks the National Science Foundation (grant DMS-0203991). The second and third authors were partially supported by the National Science Council, Republic of China, under contract nos. NSC94-2811-M-110-016 and NSC94-2115-M-110-011. 


\section{REFERENCES}

1. M.S. Ashbaugh and R.D. Benguria, Optimal bounds for ratios of eigenvalues of onedimensional Schrödinger operators with Dirichlet boundary conditions and positive potentials, Comm. Math. Phys., 124 (1989) 403-415. MR1012632 (91c:34114)

2. D.C. Barnes, The inverse eigenvalue problem with finite data, SIAM J. Math. Anal., 22 (1991) 732-753. MR1091678 (91k:34016)

3. Y.T. Chen, Y.H. Cheng, C.K. Law and J. Tsay, $L^{1}$ convergence of the reconstruction formula for the potential function, Proc. Amer. Math. Soc., 130 (2002) 2319-2324. MR1896415 (2002k:34021)

4. Y.H. Cheng, C.K. Law and J. Tsay, Remarks on a new inverse nodal problem, J. Math. Anal. Appl., 248 (2000) 145-155. MR.1772587 (2001c:34027)

5. O.H. Hald and J.R. McLaughlin, Solutions of inverse nodal problems, Inverse Problems, 5 (1989) 307-347. MR.999065 (90c:34015)

6. O.H. Hald and J.R. McLaughlin, Inverse problems: recovery of BV coefficients from nodes, Inverse Problems, 14 (1998) 245-273. MR:1619441 (99b:34028)

7. A. Kirsch, An Introduction to the Mathematical Theory of Inverse Problems, Springer-Verlag, New York, 1996. MR1479408 (99c:34023)

8. C.K. Law, C.L. Shen and C.F. Yang, The inverse nodal problem on the smoothness of the potential function, Inverse Problems, 15 (1999), 253-263; Errata, 17 (2001) 361-364. MR.1675348 (2000a:34020) MR 1838233 (2002d:34020)

9. C.K. Law and J. Tsay, On the well-posedness of the inverse nodal problem, Inverse Problems, 17 (2001) 1493-1512. MR.1862204 (2002g:34024)

10. C.K. Law and C.F. Yang, Reconstructing the potential function and its derivatives using nodal data, Inverse Problems, 14 (1998), 299-312. MR1619374 (99b:34029a)

11. J.R. McLaughlin, Inverse spectral theory using nodal points as data-a uniqueness result, J. Diff. Eqns., 73 (1988) 354-362. MR943946 (89f:34035)

12. C.L. Shen, On the nodal sets of the eigenfunctions of the string equation, SIAM J. Math. Anal., 6 (1988) 1419-1424. MR965261 (89j:34035)

13. X.F. Yang, A solution of the inverse nodal problem, Inverse Problems, 13 (1997) 203-213. MR 1435878 (98c:34017)

14. X.F. Yang, A new inverse nodal problem, J. Diff. Eqns., 169 (2001) 633-653. MR.1808480 (2001m:34063) 15260

Department of Mathematics, University of Pittsburgh, Pittsburgh, Pennsylvania

E-mail address: xinfu@pitt.edu

Department of Applied Mathematics, National Sun Yat-sen University, Kaohsiung 804, TAIWAN

E-mail address: jengyh@math.nsysu.edu.tw

Department of Applied Mathematics, National Sun Yat-Sen University, Kaohsiung 804, Taiwan - And - National Center for Theoretical Sciences, Taiwan

E-mail address: law@math.nsysu.edu.tw 